\title{
AN EXAMPLE CONCERNING AFFINE CONNEXIONS
}

\section{N. HICKS}

Introduction. The purpose of this note is to exhibit a simply connected manifold on which is defined a complete affine connexion with the following property: for each integer $n>0$ there are two points such that any broken geodesic between them must contain at least $n$ breaks. The idea is to take a manifold, say $M$, with complete affine connexion such that the exponential map is not onto, remove neighborhoods of two points, getting $M^{\prime}$, so that any geodesic (in $M$ ) leaving one of these neighborhoods must break at least once in order to intersect the other neighborhood, then attach a countable number of copies of $M^{\prime}$ together through these neighborhoods. The difficulty arises in accomplishing the attaching while preserving completeness. Perhaps this process may prove useful in other studies. This example shows that Theorem 1 in [3] (see bibliography) does not hold if a bound is placed on the number of breaks a broken geodesic may have.

The author would like to thank M. Berger, I. M. Singer, and W. Kaplan for helpful suggestions.

1. Local flattening of a connexion. Let $M$ be a $C^{\infty}$ manifold on which is defined a complete affine connexion $\omega$, i.e., a connexion on the bundle of bases $B(M)$ (see [1]). Take a point $m$ in $M$ and let $\phi$ be a coordinate map with coordinate functions $x_{1}, \cdots, x_{n}$ on a neighborhood $U$ of $m$. We take $U$ so that the exponential map (for $\omega$ ) at $m$ is a diffeo with respect to $U$ and $U$ is convex (see [4]). Let $\Gamma$ denote the set of functions $\Gamma_{j k}^{t}$ belonging to $\omega$ and $\phi$; we assume $\phi$ is chosen so that all $\Gamma_{j k}^{i}(m)=0$. Further we suppose all $x_{i}(m)=0$ and $p$ in $U$ implies $\sum_{1}^{n} x_{2}(p)^{2}<R^{2}$. Let $d$ denote the distance function in $U$ induced by this coordinate system. For $0<r<R$, let $U(r)$ be the open ball about $m$ of radius $r$. In general, let $B(p, r)$ $=[q \in U: d(p, q)<r]$.

THEOREM 1. There is a complete connexion $\bar{\omega}$ on $M$ and a real number $s$ such that: (1) $0<s<R$, (2) $\omega=\bar{\omega}$ on $M-U(s)$, (3) $\bar{\omega}$ is the flat Riemannian connexion associated with $d$ in $U(s / 2)$.

PRoof. For each $r, 0<r<R$, let $f_{r}$ be a real valued $C^{\infty}$ function on $M$ with $f_{r}(p)=0$ for $p$ in $U(r / 2), f_{r}(p)=1$ for $p$ in $M-U(r)$, and $0 \leqq f_{r} \leqq 1$. Moreover we may assume the mapping: $(r, p) \rightarrow f_{r}(p)$ is continuous on the set $(0, R) \times M$. Let $f_{0} \equiv 1$ on $M$. For each $r, 0 \leqq r<R$,

Received by the editors December 17, 1959. 
let $\omega_{r}$ be the connexion on $M$ defined by requiring $\omega_{r}=\omega$ on $M-U$, while $\Gamma_{r}=f_{r} \Gamma$ defines $\omega_{r}$ in terms of the coordinate map $\phi$ in $U$.

Note the function: $(r, p) \rightarrow f_{|r|}(p) \Gamma(p)$ is continuous on $(-R, R) \times U$. This is trivial at points where $r \neq 0$. For a point $(0, p)$ take any $\epsilon>0$. If $p=m$, let $\delta>0$ be chosen so that $q$ in $U(\delta)$ implies $\left|\Gamma_{j k}^{t}(q)\right|<\epsilon$ for all $i, j, k$. Then if $(r, q)$ in $(-R, R) \times U(\delta),\left|f_{|r|}(q) \Gamma(q)-0\right|<\epsilon$. If $p \neq m$, let $\delta_{1}=d(m, p) / 2$. Let $\delta_{2}>0$ be chosen so that $q$ in $B\left(p, \delta_{2}\right)$ implies $\left|\Gamma_{j k}^{t}(q)-\Gamma_{j k}^{\prime}(p)\right|<\epsilon$ for all $i, j, k$, and $\delta_{2}<\delta_{1}$. Then if $(r, q)$ in $\left(-\delta_{1}, \delta_{1}\right) \times B\left(p, \delta_{2}\right),\left|f_{|r|}(q) \Gamma(q)-\Gamma(p)\right|=|\Gamma(q)-\Gamma(p)|<\epsilon$ since $q$ in $M-U\left(\delta_{1}\right)$.

Let $T(M)$ be the tangent bundle to $M$; let $\pi: T(M) \rightarrow M$; let $W=\left[(p, X)\right.$ in $T(M): p$ in $U(R / 2)$ and $\exp _{m}( \pm X)$ in $\left.U(R / 2)\right]$; let $y_{i}=x_{i} \circ \pi$ and $y_{n+i}=d x_{i}$ for $i=1, \cdots, n$. Then the differential equations for "lifted" geodesics for the $\omega_{r}$ connexion become (the prime denote differentiation with respect to $t$ ),

$$
\left(y_{i}\right)^{\prime}=y_{n+i}, \quad\left(y_{n+i}\right)^{\prime}=-\sum_{j k}\left(f_{r} \Gamma_{j k}^{i} \circ \pi\right) y_{n+j} y_{n+k}
$$

summing $j$ and $k$ from 1 to $n$. We abbreviate this system by writing $y^{\prime}=F(y, r)$. Note $F$ is a continuous function on $D=W \times(-R / 2, R / 2)$ and satisfies a Lipschitz condition in $y$ uniformly on $D$.

Use the notation $g(t,(p, X), r)$ to denote a geodesic of the $\omega_{r}$ connexion defined on some interval about $t=0$, with $0 \rightarrow p$ and tangent vector $X$ at $t=0$. We know $g(t,(p, X), 0)$ is defined for all $t$ by completeness of $\omega$, but the image points may not all remain in $U$.

For each $(p, X)$ in $W$ we know $g(t,(p, X), 0)$ is defined and stays in $U$ for $t$ in $[-1,1]$. A slight variation of a theorem from the theory of differential equations (see $\left[2\right.$, p. 29]) gives us a $\delta_{1}>0$ such that if $|r|<\delta_{1}$ then $g(t,(p, X), r)$ is defined for $t$ in $[-1,1]$. Now let

$$
W^{\prime}=\left[(p, X) \text { in } W: \frac{3 R}{8} \leqq d\left(m, \exp _{p} \frac{1}{2} X\right) \leqq \frac{R}{2}\right] .
$$

Let $V=U(R / 8)$, let $W^{\prime \prime}=W^{\prime} \cap \pi^{-1}(\bar{V})$. Hence $W^{\prime \prime}$ is compact. The theorem from differential equations assures us $g(t,(p, X), r)$ is continuous on the set $(-1,1) \times W \times\left(-\delta_{1}, \delta_{1}\right)$ and hence is uniformly continuous on $A=[-1 / 2,1 / 2] \times W^{\prime \prime} \times\left[-\delta_{1} / 2, \delta_{1} / 2\right]$. Thus for the number $R / 16$ we obtain $\delta_{2}>0$ such that if we have elements $\left(t_{1},\left(p, X_{1}\right), r_{1}\right)$ and $\left(t_{2},\left(p_{2}, X_{2}\right), r_{2}\right)$, both in $A$, with $\left|t_{1}-t_{2}\right|<\delta_{2}$, $d\left(\left(p_{1}, X_{1}\right),\left(p_{2}, X_{2}\right)\right)<\delta_{2}$ and $\left|r_{1}-r_{2}\right|<\delta_{2}$, then

$$
d\left(g\left(t_{1},\left(p_{1}, X_{1}\right), r_{1}\right), g\left(t_{2},\left(p_{2}, X_{2}\right), r_{2}\right)\right)<\frac{R}{16} .
$$


Now take $s$ such that $0<s<\operatorname{minimum}\left[\delta_{1} / 2, \delta_{2}, R / 8\right]$. Claim $\bar{\omega}=\omega_{s}$ is the desired connexion. We need only show $\bar{\omega}$ is complete.

Since $\bar{\omega}=\omega$ in $M-U(s)$ we need only worry about geodesics emanating from (or passing through) $U(s)$. Take $p$ in $U(s)$ and take any $Y$ tangent to $M$ at $p$. There is a real number $b$ such that $(p, b Y)$ in $W^{\prime \prime}$, i.e., $3 R / 8 \leqq d\left(m, \exp _{p} \frac{1}{2} b Y\right) \leqq R / 2$, for $U$ is convex with respect to the $\omega$ connexion. Then $g(t,(p, b Y), s)$ is defined on $[-1,1]$ and for $t=1 / 2$ we claim $d(m, g(\alpha))>s$ where $\alpha=(1 / 2,(p, b Y), s)$, hence $g$ has reached a point at which $\bar{\omega}=\omega$ and hence may be indefinitely extended. The above inequality follows by letting $\beta=(1 / 2,(p, b Y), 0)$, hence $\alpha$ and $\beta$ are both in $A$ and within $\delta_{2}$ of each other which implies $d(g(\alpha), g(\beta))<R / 16$. But $d(m, g(\beta)) \geqq 3 R / 8$, hence

$$
d(m, g(\alpha)) \geqq \frac{3 R}{8}-\frac{R}{16}=\frac{5}{16} R>\frac{R}{8}>s . \quad \text { q.e.d. }
$$

We remark if we take $\bar{\omega}=\omega_{8^{\prime}}$ for any $0<s^{\prime}<s$ the conclusion of Theorem 1 still holds.

2. Attaching two spaces. Let $M$ and $M^{\prime}$ be two $C^{\infty}$ manifolds, with the same dimension $n$, each carrying complete connexions. We wish to define a process which can roughly be described as follows. We remove two particular neighborhoods $V$ and $V^{\prime}$ from $M$ and $M^{\prime}$ respectively. Let $P=(M-V) \cup N \cup\left(M^{\prime}-V^{\prime}\right)$ where $N$ is a "neck" attached to $M-V$ along the boundary of $V$ and similarly attached to $M^{\prime}-V^{\prime}$. We then define a complete connexion on $P$ which coincides with the original connexions in $M-V$ and $M^{\prime}-V^{\prime}$.

Using the function $\exp \left(-(1 / x)^{2}\right)$ we may construct a regular univalent $C^{\infty}$ function $\phi: E^{1} \rightarrow E^{2}$ such that for $t \geqq 1, \phi(t)=(t, 0)$; for $t \leqq-1, \phi(t)=(-t, 1)$; moreover, letting $\phi(t)=(x(t), y(t))$ we assume for $-1<t<1$ that $y(t)$ is strictly decreasing with $0<y(t)<1$; for $0<t<1, x(t)$ is strictly decreasing with $1 / 2<x(t)<1, x(0)=1 / 2$; and $x(-t)=x(t)$ for $-1<t<0$.

For any real number $r>0$ we define $N(r)$ to be the $n$-dimensional regular submanifold of $E^{n+1}$ consisting of the union of the three sets:

$$
\begin{aligned}
U_{0} & =\left[\left(a_{1}, \cdots, a_{n}, 0\right): \sum_{1}^{n} a_{i}^{2} \geqq r^{2}\right], \\
U_{1} & =\left[\left(a_{1}, \cdots, a_{n}, 1\right): \sum_{1}^{n} a_{i}^{2} \geqq r^{2}\right], \\
U_{2} & =\left[\left(a_{1} x(t), \cdots, a_{n} x(t), y(t)\right): \sum_{1}^{n} a_{i}^{2}=r^{2} \text { and }-1 \leqq t \leqq 1\right] .
\end{aligned}
$$


Using the Riemannian metric on $N(r)$ induced from the Euclidean metric on $E^{n+1}$, we obtain a complete connexion $\omega_{0}$ on $N(r)$ since $N(r)$ is complete (closed) as a subset of $E^{n+1}$.

Let $M$ and $M^{\prime}$ be $C^{\infty}$ manifolds of dimension $n$ carrying complete affine connexions $\omega$ and $\omega^{\prime}$, respectively. Let $m, m^{\prime} ; U, U^{\prime} ; x_{i}, x_{i}^{\prime}$; $R, R^{\prime}$; and $d, d^{\prime}$ all be chosen as in $\S 1$ for $M$ and $M^{\prime}$ respectively. Applying Theorem 1 to $M$ and $M^{\prime}$ we obtain a connexion $\bar{\omega}$ on $M$ and number $s$, and a connexion $\bar{\omega}^{\prime}$ on $M^{\prime}$ and number $s^{\prime}$. By taking the smaller we may assume $s=s^{\prime}$ by the remark at the end of $\$ 1$. Let $V=U(s / 4), V^{\prime}=U^{\prime}(s / 4)$. Let $N(s / 4)$ be the submanifold of $E^{n+1}$ defined above. Let $N=\left[p\right.$ in $N(s / 4): p$ in $\left.U_{2}\right]$. Let $P=(M-V) \cup N$ $\cup\left(M^{\prime}-V^{\prime}\right)$. If $p$ is in the boundary of $V$ we identify $p$ with the point in $N$ with coordinates $\left(x_{1}(p), \cdots, x_{n}(p), 0\right)$; similarly, for $p^{\prime}$ in the boundary of $V^{\prime}$ we identify $p^{\prime}$ with the point in $N$ with coordinates $\left(x_{1}^{\prime}(p), \cdots, x_{n}^{\prime}\left(p^{\prime}\right), 1\right)$. Our definition of $N$ assures us $P$ is a $C^{\infty}$ manifold. Let $\varpi$ be the connexion defined on $P$ by $\varpi=\bar{\omega}$ on $M-V$, $\varpi=\omega_{0}$ on $N, \varpi=\bar{\omega}^{\prime}$ on $M^{\prime}-V^{\prime}$. By Theorem 1 these connexions agree on the "overlap." Furthermore $\varpi$ is complete and $\varpi=\omega$ on $M-U(s)$, $\varpi=\omega^{\prime}$ on $M^{\prime}-U^{\prime}(s)$.

We refer to the above process by saying we have attached $M$ and $M^{\prime}$ through the neighborhoods $U$ and $U^{\prime}$ respectively.

3. The example. Let $G$ be the Lie group $S L(2, C)=[2 \times 2$ matrices $T$ over $C$ : $\operatorname{det} T=1]$. Let $\omega$ be the complete connexion on $G$ defined by left translation. We provide two points $p_{1}$ and $p_{2}$ in $G$, with neighborhoods $U_{1}$ and $U_{2}$ respectively, such that any geodesic leaving $U_{1}$ must break at least once in order to intersect $U_{2}$. Then going to the simply connected covering and "inverting" through these neighborhoods we obtain the example.

We recall that the exponential map from the Lie algebra of $G$ into $G$ does not map onto $G$, for any element in the image has a square root and any element in this group having a square root has trace $\geqq-2$, however there exist elements with trace $<-2$. Let $p$ be in $G$ with trace $p<-2$, let $e$ be the identity. Since trace is continuous we may let $U$ be a neighborhood of $p$ such that trace $(U)<-2$. We further take $U=V_{1} p$ where $V_{1}$ is a neighborhood of $e$. Let $V$ be a neighborhood of $e$ with $V V \subset V_{1}$ and $V=V^{-1}$. We claim no unbroken geodesic emanating from a point in $V$ intersects $V p$. For if $g$ was such a geodesic, say $g(0)=m$ in $V, g(a)=p^{\prime}$ in $V p$, then $m^{-1} g(0)=e$, $m^{-1} g(a)=m^{-1} p^{\prime}=m^{-1} m^{\prime} p$ is in $U$. But $m^{-1} g$ is a geodesic and no unbroken geodesic passing through $e$ can intersect $U$ because of the trace condition. Let $U_{1}=V, U_{2}=V p$.

For each integer $n \geqq 1$, let $M_{n}=G$. By $\S 2$ we may attach each $M_{k}$ 
to $M_{k+1}$, for $k \geqq 1$, through the neighborhoods $U_{2}$ and $U_{1}$ respectively, obtaining a manifold $N$ with complete connexion which agrees with $\omega$ on each $M_{k}-\left(U_{1} \cup U_{2}\right), k>1$. Hence for any integer $n$ we need only let $p_{0}=e$ in $M_{1}-U_{2} \subset N$ and let $p_{n}$ be any point in $M_{n}-\left(U_{1} \cup U_{2}\right)$, then any broken geodesic from $p_{0}$ to $p_{n}$ must break at least $n$ times. Finally, let $M$ be the simply connected covering of $N$, let $\pi: M \rightarrow N$ be the covering map, and define a connexion $\omega^{*}$ on $M$ by $\omega^{*}=\pi^{*} \omega$. For each $i \geqq 0$, let $m_{i}$ be a point in $M$ with $\pi\left(m_{i}\right)=p_{i}$. Then any broken geodesic from $m_{0}$ to $m_{n}$ must contain at least $n$ breaks since $\pi$ carries a geodesic in $M$ into a geodesic in $G$.

\section{BibLIOGRAPHY}

1. W. Ambrose and I. M. Singer, $A$ theorem on holonomy, Trans. Amer. Math. Soc. vol. 75 (1953) pp. 428-443.

2. E. A. Coddington and N. Levinson, Theory of ordinary differential equations, New York, McGraw-Hill, 1955, p. 29.

3. N. Hicks, $A$ theorem on affine connexions, Illinois J. Math. vol. 3 (1959) pp. 242-254.

4. J. H. C. Whitehead, Convex regions in the geometry of paths, Quart. J. Math. (Oxford Ser.) vol. 3 (1932) pp. 33-42.

The University of Michigan 